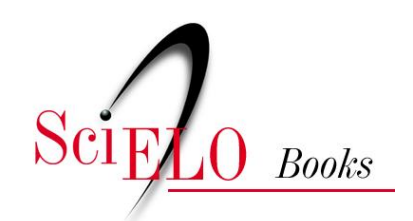

\title{
Una nueva mirada hacia la ética en la sexualidad y su impacto en la sociedad actual
}

\author{
José Miguel Peláez Freire \\ Jeverson Santiago Quishpe Gaibor \\ José Eduardo Paucar Paucar
}

\section{SciELO Books / SciELO Livros / SciELO Libros}

PELÁEZ FREIRE, J.M., QUISHPE GAIBOR, J.S., and PAUCAR PAUCAR, J.E. Una nueva mirada hacia la ética en la sexualidad y su impacto en la sociedad actual. In: IZA VILLACÍS, V.A., ed. Persona, educación y filosofia: reflexiones desde la educación universitaria [online]. Quito: Editorial Abya-Yala, 2018, pp. 105-122. ISBN: 978-9978-10-493-4.

https://doi.org/10.7476/9789978104934.0007.

International license.

Todo o conteúdo deste trabalho, exceto quando houver ressalva, é publicado sob a licença Creative Commons Atribição 4.0.

Todo el contenido de esta obra, excepto donde se indique lo contrario, está bajo licencia de la licencia $\underline{\text { Creative }}$ Commons Reconocimento 4.0. 


\title{
Una nueva mirada hacia la ética en la sexualidad y su impacto en la sociedad actual
}

\author{
José Miguel Peláez Freire ${ }^{1}$ \\ Jeverson Santiago Quishpe Gaibor ${ }^{2}$ \\ José Eduardo Paucar Paucar ${ }^{3}$
}

\section{Resumen}

En la actualidad la sociedad está llena de ideas liberales que dominan el comportamiento de las personas, ocasionando muchas veces una incorrecta comprensión de la sexualidad y una tendencia a confundir la ética con moralismo. Lo bueno y lo malo no depende de nuestras valoraciones subjetivas o sociales, sino que cada persona es consciente de lo que es bueno y malo, por ende, existen conductas sexuales buenas y otras malas; además, no se debe considerar a la ética ligada a creencias dogmáticas o religiosas, sino como algo independiente.

1 Magister en Gerencia Educativa. Magister en Teología Civil. Doctorando de PhD Teología Civil. Docente de la Carrera Ciencias Administrativas, Universidad Politécnica Salesiana de Guayaquil. jpelaez@ups.edu.ec

2 Doctorando de $\mathrm{PhD}$ Teología Civil. Docente de la Carrera de Pedagogía, Universidad Politécnica Salesiana de Quito. jquisphez@ups.edu.ec

3 Magíster en Gestión Educativa. Doctorando de PhD Teología Civil. Docente de la Carrera de Biotecnología de los Recursos Naturales. Universidad Politécnica Salesiana de Quito. jpaucar@ups.edu.ec 
$\mathrm{Al}$ igual que cada función que tiene nuestro cuerpo la sexualidad tiene sus funciones que no podríamos decir a ciencia cierta que es buena o mala, depende de cómo cada persona la utilice, por ello el determinar qué es ético y qué no, dentro de la sexualidad depende de la formación que se haya obtenido. Si hablamos de conductas que no son éticas dentro de la sexualidad podríamos mencionar temas como; el abuso sexual, la violencia sexual, el exhibicionismo con fines comerciales, todo tipo de engaño o victimización, la utilización de la sexualidad de una forma no responsable con riesgo para uno mismo o para nuestra pareja, el ejercicio del poder a través de la sexualidad, la enseñanza de una sexualidad distorsionada, irreal y mentirosa.

Es importante reflexionar sobre este tema teniendo como base el amor, la responsabilidad, la confianza mutua, el diálogo y por supuesto la acción más trascendente e importante que nos da la sexualidad para una mejor valoración personal, familiar y social.

Palabras claves: Ética, sexualidad, sociedad, desafíos.

\section{Abstract}

At present, society is full of liberal ideas that dominate the behavior of people, often causing an incorrect understanding of sexuality and a tendency to confuse ethics with moralism. The good and the bad do not depend on our subjective or social evaluations, but each person is aware of what is good and bad, therefore, there are good and bad sexual behaviors; In addition, ethics linked to dogmatic or religious beliefs should not be considered, but rather as something independent.

Just like every function that our body has, sexuality has its functions that we can not say with certainty that it is good or bad, it depends on how each person uses it, therefore, determining what is ethical and what is not, within sexuality it depends on the training that has been obtained. If we talk about behaviors that are not ethical within sexuality we could mention topics such as; sexual abuse, sexual violen- 
ce, exhibitionism for commercial purposes, all kinds of deception or victimization, the use of sexuality in a non-responsible way with risk for oneself or for our partner, the exercise of power through sexuality, the teaching of a distorted, unreal and deceitful sexuality.

It is important to reflect on this theme based on love, responsibility, mutual trust, dialogue and, of course, the most transcendent and important action that sexuality gives us for a better personal, family and social assessment.

Keywords: Ethics, sexuality, society, challenges.

\section{Introducción}

La sexualidad es un elemento básico de la personalidad; un modo propio de ser, de manifestarse, de comunicarse con los otros, de sentir, expresar y vivir el amor humano. Todas las personas somos intrínsecamente sexuales, y el desarrollo sexual evoluciona durante la infancia y la adolescencia, manifestándose durante el resto de las etapas de la vida. Pareciera que el ejercicio de la sexualidad es un aspecto que solo impacta en la persona en lo individual; sin embargo, el ejercicio de la sexualidad tiene efectos biológicos, psicológicos, sociales y espirituales; por lo que para tener una sociedad sana y productiva es necesario promover la salud sexual, porque sexualidad y el bienestar sexual son componentes integrales de la salud y el desarrollo (López Trujillo, 2013, p. 27).

El autor en la cita expuesta presenta a la sexualidad como parte fundamental en el desarrollo del ser humano, pone en evidencia que la sexualidad humana es parte esencial de la personalidad, hace que ver que el ser humano es un ser sexuado y como tal se manifiesta en todas las facetas de la vida. Si bien es cierto, la sociedad impone normas morales que inciden en el comportamiento sexual de las personas, en última instancia es la persona quien asume la responsabilidad de sus actos; y aquí entra en juego la formación que se haya recibido. Pues la sexualidad es una dimensión fundamental 
en cada ser humano y no únicamente una necesidad biológica, por lo tanto, la sexualidad tiene que ser vivida con responsabilidad, porque toca a todo el ser humano en su integralidad; lo biológico, psíquico y espiritual tienen que armonizarse en una sexualidad que tiene como fundamento el amor. En este sentido, la vivencia de una sexualidad responsable en pareja tiene que conducir al cuidado mutuo, al respeto, a la reciprocidad y a tener claras las consecuencias.

El estado por su parte debe promover una cultura de respeto a los derechos sexuales y el acceso a los servicios de salud sexual y reproductiva; pero más que un respeto por los derechos sexuales debe suscitar una educación correcta y oportuna en donde se pueda brindar la información apropiada para que los jóvenes en la actualidad tomen conciencia de sus derechos pero a la vez de sus responsabilidades con respecto a la sexualidad, la orientación oportuna es importante ya que la sexualidad no es un juego, al mismo tiempo es muy necesario que la sociedad como tal deje los tabúes que existen a la hora de hablar de este tema en los colegios, en las universidades, principalmente en los hogares a fin de evitar embarazos precoces, abortos, enfermedades y demás consecuencias que se puedan presentar a causa de una mala información y orientación.

\section{Metodología}

La metodología empleada fue una sistematización teórica realizada a través de la consulta a fuentes bibliográficas como libros, periódicos y documentos de instituciones, se empleó el análisis y la síntesis, la inducción y la deducción, para el procesamiento de la información.

\section{Desarrollo}

El amor, que se alimenta y se expresa en el encuentro del hombre y de la mujer, es don de Dios; es por esto fuerza positiva, orientada a su madurez en cuanto personas; es a la vez una preciosa reserva para el don de sí que todos, hombres y mujeres, están llamados a cumplir para su propia realización y felicidad, según un proyecto de 
vida que representa la vocación de cada uno. El hombre, en efecto, es llamado al amor como espíritu encarnado, es decir, alma y cuerpo en la unidad de la persona. El amor humano abraza también el cuerpo y el cuerpo expresa igualmente el amor espiritual. La sexualidad no es algo puramente biológico, sino que mira a la vez al núcleo íntimo de la persona. El uso de la sexualidad como donación física tiene su verdad y alcanza su pleno significado cuando es expresión de la donación personal del hombre y de la mujer hasta la muerte. Este amor está expuesto, sin embargo, como toda la vida de la persona, a la fragilidad debida al pecado original y sufre, en muchos contextos socio-culturales, condicionamientos negativos y a veces desviados y traumáticos. Sin embargo la redención del Señor, ha hecho de la práctica positiva de la castidad una realidad posible y un motivo de alegría, tanto para quienes tienen la vocación al matrimonio -sea antes y durante la preparación, como después, a través del arco de la vida conyugal-, como para aquellos que reciben el don de una llamada especial a la vida consagrada (López Trujillo, 2013, p. 61).

Aquí el autor subraya la centralidad del amor en la vivencia de la sexualidad, que hace que el hombre y la mujer alcancen la plena realización como fruto de la donación y entrega total en cuanto seres llamados al amor. Desde la perspectiva de fe, esta experiencia vital de entrega mutua tiene su fundamento último en Dios que es la fuente del amor, de allí que el amor y todas sus expresiones manifestadas en la relación de pareja es un don de Dios. Sin embargo, la vivencia profunda del amor puede verse afectada por la fragilidad y las limitaciones humanas que ponen al centro el egoísmo, la maldad, los caprichos y las pasiones hedonistas, desvirtuando de este modo el precioso don de la sexualidad.

La sexualidad es mucho más que algo biológico, la educación ética, pretende brindar unas condiciones para cumplir las dos funciones: ser felices y llevar una convivencia digna. Esta ética de la sexualidad debe incluir una formación de la libertad y de los afectos, donde se priorice lo espiritual a lo meramente material, los valores 
a los impulsos, lo social a lo individualista, el bien común al interés particular, el amor al deseo, e incluso, la abstinencia sexual a las técnicas anticonceptivas como "cuidado" de las consecuencias del ejercicio de la sexualidad.

La liberación sexual del presente siglo ha favorecido la superación del dualismo cuerpo-alma en la comprensión de la sexualidad humana. Se desarticula así la visión dicotómica, estereotipada y simplificada de la sexualidad que la identificaba con cuerpo-carne- irracionalidad-mundo animal-lo intrascendente-inferior efímero-malo y separada del alma -del espíritu-de lo humano-de lo racional y trascendente. Uno de los logros inestimable de este siglo que concluye, es la valoración de la sexualidad como aspecto importante de la personalidad humana (Fernández, 2004).

Entonces la sexualidad no es solamente un acto físico ligado al placer, sino que va más allá de eso, porque es una entrega de sí mismo, de cuerpo, mente y espíritu. Es parte de cada ser humano y es fundamental que le demos la valoración que merece, pensar en esta como un acto racional que debe ser tomado con responsabilidad. No podemos considerarla como algo momentáneo o pasajero, por lo que debe ser realizada con una persona con la que nos sintamos estrechamente relacionados, ya que la sexualidad es una pieza esencial en el desarrollo de nuestra personalidad, de ser valorada como un acto hedonista al final nos provocará un vacío espiritual.

La sexualidad permite expresar libremente todo nuestro ser, que involucra factores biológicos, psicológicos, sociales, culturales y espirituales. Dando a entender que cada persona es un mundo diferente que se manifiesta a través de la sexualidad con un propio modo de ser, de sentir, de comunicar y de reflejar el amor; por lo que tenemos el derecho de poder decidir con quién compartir nuestra vida y sexualidad.

El estudio del asertividad sexual ha generado resultados que demuestran su importancia y su papel fundamental en la sexualidad humana. En este estudio teórico se lleva a cabo una revisión sistemá- 
tica de los principales resultados obtenidos en estos estudios. Después de una búsqueda en las principales bases de datos se obtiene un total de 76 trabajos publicados entre 1980 y 2009, que demuestran que la asertividad sexual es un factor determinante tanto de la respuesta sexual como del funcionamiento sexual humano. Además, se relaciona de forma directa con una visión positiva de la sexualidad humana y con algunas variables sociodemográficas como el sexo, aunque esta relación no está clara. Otros estudios ponen de manifiesto que es un factor de protección ante experiencias de abuso y victimización sexual, así como ante conductas sexuales de riesgo. Se discuten los resultados y se plantea la necesidad de incluir el asertividad sexual de forma específica, más que el asertividad general, en los programas educativos y en intervenciones con poblaciones en situación de riesgo (Santos, 2010).

Este estudio complementa la reflexión, muestra la importancia de la asertividad sexual dentro de un contexto social y psicológico donde el ser humano logra una completa satisfacción en su relación junto con la persona que lo haga sentir íntegro y completo a la hora de llevar a cabo un acto sexual físico o a su vez la interrelación sexual de ser humano. También se toma en cuenta que esta medida de búsqueda acertada en la comunicación sexual es un mecanismo de defensa, de todos los abusos y daño causado por un error de comunicación sexual o emocional conocidas como conductas sexuales de riesgo.

La sexualidad forma parte de nuestro comportamiento, es un elemento más de nuestra libertad. La sexualidad es obra nuestra es una creación personal y no la revelación de aspectos secretos de nuestro deseo. A partir y por medio de nuestros deseos, podemos establecer nuevas modalidades de relaciones, nuevas modalidades amorosas y nuevas formas de creación. "El sexo no es una fatalidad, no; es una posibilidad de vida creativa” (Foucault, 1982).

La sexualidad como parte fundamental para una vida plena y saludable donde el ser humano se mide esencialmente por su deseo de llevar cabo un acto sexual cargado de nuevo significado y sentido, 
que le conduzca a la realización, al ejercicio pleno de la libertad, a potenciar su estilo de vida. A través de los deseos, adecuadamente orientados y canalizados, el ser humano puede generar creativamente nuevas formas y modalidades de encuentro íntimo con la pareja. Todo esto tiene sentido, siempre y cuando se busca el bien y la realización del otro. Entonces el sexo se convierte en un don, en una posibilidad permanente de renovación, en una nueva oportunidad de ser feliz auténticamente y de ser portador de vida.

La sexualidad es, evidente, una de las virtudes concupiscibles, ya que busca el placer y tiende a la evitación del dolor. Esto es natural, y en tanto que natural es bueno. Pero las virtudes éticas solo son moralmente correctas cuando se hallan bajo el imperio de la razón. Las virtudes éticas dependen siempre de las dianoéticas. Sin ellas serían puramente irracionales, y por tanto no serían virtudes morales en el sentido estricto de la palabra. De ahí que, aunque el ejercicio de la sexualidad sea una función natural del organismo, y aunque sea bueno y natural buscar el placer y evitar el dolor, esto no será humano y moral más que cuando se halle controlado por la razón, es decir, cuando no se haga de modo puramente instintivo o animal, sino humano racional (Gracia, 1998).

Este concepto interesante sobre la ética en la sexualidad de la vida de las personas permite revalorar que nosotros los seres humanos nacemos sexuados y nuestra naturaleza biológica nos impulsa a asociarnos y compartir sentimientos y pensamientos con las personas que nos rodean. Además, debemos comprender que la sexualidad forma parte del ser y que el ejercicio de la misma, si bien es también una experiencia biológica y placentera, no se queda condicionada a ese plano, sino que se reviste de algo más profundo que es la capacidad reflexiva, analítica, racional; en otras palabras, la sexualidad alcanza profundidad cuando es vivida con inteligencia y, más allá, con sabiduría. Esta experiencia vital alcanza su culmen en la conexión espiritual a la que pueden llegar los seres humanos en el abrazo sexual y de este modo, alcanzar la plena felicidad. 
En la ética naturalista de la sexualidad, los actos son intrínsecamente buenos cuando respetan el orden interno de la naturaleza e intrínsecamente malos cuando no lo respetan. Además, la sexualidad se hace moral cuando se ejercita con moderación. Para el caso de los órganos de la generación, sus fines son la reproducción y perpetuación de la especie, de tal manera que los usos no dirigidos a ellos serían inmorales. La virtud que acompaña el ejercicio de la vida sexual es la templanza, el justo medio aristotélico; por consiguiente, el exceso o abstinencia serían inmorales.

Como la sexualidad es, en esencia, una actividad privada, "un acto será moralmente reprobable cuando viole la autonomía de las personas o éstas no lo consideren beneficioso para ellas" (Montoya, 2007, p. 58).

Por esta razón la sexualidad hoy en día necesita de la moralidad y la ética para que la sociedad comprenda que este concepto no solamente se basa en el sexo, sino que más bien la sexualidad nos ayuda a relacionarnos con las demás personas ya sea hombre o mujer en un sentido amplio de afectividad y sentimientos hacia el otro ser con el fin de vivir en armonía y felicidad. El ejercicio de la sexualidad conlleva a conocer el comportamiento de ambos sexos, conocer las necesidades del uno y del otro tanto físicas como emocionales; conlleva entrar en comunión con el otro y comprender y aceptar sus valores, sus proyectos, sus sueños, sus ideales y esperanzas.

La propia evolución humana en cuanto al conocimiento científico, la cultura y reflexión han generado el debilitamiento y abandono de normas erigidas sobre presupuestos hoy inadmisibles y que hasta entonces habían regulado el comportamiento sexual de modo dominante y restrictivo. Así, ante una sexualidad vista como pecado, vergüenza asociada a la reproducción, al matrimonio y a la moral convencional, se impone cada vez con más fuerza la sexualidad como valor, placer, comunicación, ternura, dimensión de lo personal y la forma más profunda de encuentro interpersonal humano. Se des institucionaliza el sexo y se impulsa un referente ético personal tendiente a una moral democrática, autónoma y responsable. Cada vez es más fuerte la repulsa a todo lo que condena o discrimina la 
sexualidad, caduca la cultura anti sexual no sin entrar por supuesto en colisión con otra cultura aún por establecerse (Rius, 2001, p. 22).

El autor presenta un correcto punto de vista del ser humano reconociéndolo como un ser sexuado, la sexualidad no implica el mero acto de una penetración sino más bien un acto de amor. Se entiende ahora que el ser humano se entrega al otro ser humano por amor y el mismo se fortalece con el compromiso, la fidelidad, la confianza y la comunión interpersonal. En el contexto actual, marcado por una fuerte tendencia hedonista, es muy importante hablar y reflexionar sobre sexualidad desde una perspectiva abierta y frontal, subrayando evidentemente el valor de la sexualidad como posibilidad de encuentro profundo, de comunión interpersonal, de potenciación de la ternura, de ser co-creadores de la vida.

En cuanto modalidad de relacionarse y abrirse a los otros, la sexualidad tiene como fin intrínseco el amor, más precisamente el amor como donación y acogida, como dar y recibir. La relación entre un hombre y una mujer es esencialmente una relación de amor: 'La sexualidad orientada, elevada e integrada por el amor adquiere verdadera calidad humana. Cuando dicho amor se actúa en el matrimonio, el don de sí expresa, a través del cuerpo, la complementariedad y la totalidad del don; el amor conyugal llega a ser, entonces, una fuerza que enriquece y hace crecer a las personas y, al mismo tiempo, contribuye a alimentar la civilización del amor; cuando por el contrario falta el sentido y el significado del don en la sexualidad, se introduce " una civilización de las "cosas" y no de las "personas"; una civilización en la que las personas se usan como si fueran cosas. En el contexto de la civilización del placer la mujer puede llegar a ser un objeto para el hombre, los hijos un obstáculo para los padres (Vaticano, 1995).

La Iglesia católica complementa y ratifica que la sexualidad tiene sentido y profundidad en cuanto está revestida de amor; el encuentro de un hombre y una mujer es fundamentalmente un encuentro de amor; sólo sobre esta base cobra sentido entonces el otro aspecto de la sexualidad que es la procreación de otro ser. Cuando una pareja decide contraer nupcias la sexualidad entra de cajón de- 
bido a que esta es un regalo de Dios, el amor conyugal hace que la familia se fortalezca y gracias a ello los hijos pueden crecer con el cariño de unos padres que se demuestran amor y respeto mutuo.

En la ética naturalista de la sexualidad, los actos son intrínsecamente buenos cuando respetan el orden interno de la naturaleza e intrínsecamente malos cuando no lo respetan. Además, la sexualidad se hace moral cuando se ejercita con moderación. La modernidad planteó una oposición entre lo natural y lo moral; el hombre pasó a ser por sí mismo la fuente de lo moral: el hombre y no la naturaleza determina lo bueno y lo malo. El ser humano se convierte en fuente de su propia moralidad, con la racionalidad como instrumento y la autonomía empieza a ser protagonista en el ámbito ético, con el respeto a la dignidad del ser humano como valor fundamental (Montoya, 2007).

En este contexto de ética y sexualidad, el ser humano como tal está lleno de principios y valores; esta ética permite la reflexión sobre la moral permitiendo encontrar el sentido a lo que nosotros somos o damos a notar frente a los demás. En el campo de la sexualidad, el criterio para determinar qué es lo bueno o qué es lo malo es el mismo ser humano en cuanto persona que está revestida de dignidad. Es bueno todo aquello que dignifica a la persona y es malo todo aquello que atenta contra la dignidad del ser humano. En la ámbito específicamente sexual, será bueno lo que conduce a la plena realización, a la vivencia del amor auténtico, a la comunión, a la entrega incondicional, al placer constructivo; por el contrario será malo lo que cosifique al otro convirtiéndolo en objeto sexual, lo que desencadena las pasiones desenfrenadas, el placer por el placer sin medida, la mercantilización del cuerpo y del sexo, le mera satisfacción de caprichos e intereses egoístas. La ética de la sexualidad tiene que ayudar a promover el respeto profundo de la dignidad del ser humano.

Cuando el encuentro sexual, en este sentido amplio del que ahora hablamos, se reduce a la superficie, permanece cautivo de las manifestaciones más externas y secundarias, o no termina, más allá de las apariencias, en el interior de la otra persona, entonces la sexualidad humana ha 
muerto. Hemos matado lo único que la vivifica, y ha sido postergada a un nivel radicalmente distinto e inferior (Azpirante, 2001).

Finalmente, cuando se habla de sexualidad en esencia se refiere a un conjunto de factores que la forman, como son el espíritu, la mente, el cuerpo, los deseos y necesidades. Cuando uno de ellos falta, deja de ser sexualidad y se convierte en algo muy simple, más simple que beber agua o dormir. Hemos desestimado tanto el uso de nuestra sexualidad y la hemos usado para fines tan básicos, que ha perdido valor e importancia. Las graves consecuencias que esto trae consigo son: embarazos no deseados, violaciones, agresión física y verbal, divorcios y en el peor de los casos la muerte. Se puede acabar con esto si empezamos a hablarle a nuestros familiares, amigos y personas cercanas de la importancia de cuidar nuestro espíritu a través del cuerpo, porque para ser felices no podemos separar ninguno de estos dos factores, somos un todo, el humano no puede clasificarse, desprenderse, o dividirse; lo que le damos al cuerpo debe ser bueno para el espíritu y viceversa, solo de ese modo podremos comprender el valor que tiene nuestra sexualidad y comprender que no podemos dejarla de lado ni borrarla de nuestro ser, porque es lo que nos identifica del resto.

La experiencia de afectividad es fundamental en la vida de los seres humanos en la medida que se establecen vínculos de encuentro y acogida profunda. Uno de los "aspectos relevantes que transversalizan todas las etapas del ciclo de vida es el vínculo afectivo, el cual tiene su expresión en la posibilidad de construir lazos o uniones de cariño, comprensión, tolerancia, aceptación, empatía, apoyo, comunicación que establecen los seres humanos" (Hernández y Sánchez, 2008, p. 56). Esto permite evidenciar que la dimensión afectiva permea toda la vida humana, sin ella no podríamos existir; pues somos seres necesitados de afecto, necesitamos de la acogida amorosa del otro para humanizarnos. Un claro ejemplo de esto es la infinita necesidad que tiene el bebé del afecto de la madre.

En determinados momentos de la historia, especialmente con el racionalismo, hubo la tendencia a relativizar lo afectivo como algo se- 
cundario cayendo incluso en su negación. Posteriores investigaciones dan cuenta de la importancia de la esfera afectiva en el ser humano.

Por ejemplo: resultan tremendamente interesantes tanto los análisis de Heidegger sobre la angustia, el aburrimiento y la maquinación como las investigaciones de Scheler sobre el sentimiento de la simpatía y el amor, pues ambos sientan las bases de lo que podemos llamar una fenomenología de los afectos que pone al descubierto los factores emocionales que animan y atraviesan la existencia humana (Escudero, 2007, p. 366).

La afectividad toca cada momento de nuestra vida y por tanto no puede ser relativizada y peor aún ignorada ya que nuestros pensamientos, sentimientos y acciones se expresan siempre con una carga afectiva. En este sentido, "no hay pensamientos ni voliciones libres de afectividad. Lo afectivo acompaña todos los actos de conocimiento y volición pues proceden de un único principio ontológico: el alma humana" (Blanco, 2013, p. 131).

Es así que los vínculos afectivos motivan a las personas a conseguir sus propósitos en el mundo educativo, empresarial, deportivo, familiar, religioso, entre otros, porque pone a los seres humanos en el plano del diálogo con el corazón, en la conexión constructiva con las emociones, sentimientos, inteligencia y su capacidad de suscitar el cambio. Evidentemente este cambio solo puede suscitarse en el equilibrio entre el mundo afectivo-emocional y la racionalidad humana, pues tenemos algo así como dos mentes: una que piensa (cabeza) y otra que siente (corazón). Las dos poseen formas distintas de saber, pero interactúan para construir nuestra vida mental integral. Regularmente las dos operan en concordancia: los sentimientos aportan información a la mente racional, mientras que los pensamientos ayudan a regular la vida emocional (Blanco, 2013, p. 133).

La interacción y equilibrio entre lo afectivo e intelectivo cobra especial importancia, por ejemplo, en el campo educativo; aquí la cognición y el afecto son esferas interactivas, que no admiten inter- 
pretaciones sectoriales, sino que todas las funciones internas generan un proceso evolutivo integrado, equilibrado e interfuncional (sensorio-perceptual, memoria, pensamiento, lenguaje, cognición, afecto, etcétera). Una tonalidad afectiva estable entre emociones y sentimientos y los procesos cognitivos genera unidad en las personas, promueve su integración como seres humanos. El vínculo afectivo es una necesidad primaria significativa que constituye la base para crear los lazos entre el individuo y su grupo social de referencia, y que sólo puede ser satisfecho dentro de la sociedad (García, 2009, p. 5).

Mirando más allá, el vínculo afectivo coloca al ser humano en el horizonte vital del amor como un camino de realización y plenificación, pues, la base sobre la que se encuentra cimentada la persona es el amor. Toda la persona halla su fundamento en el amor, pertenece a su integralidad, es por eso que el ser humano está llamado a desarrollarse siguiendo esa orientación radical de su ser (Barraca, 2005, p. 27).

La afectividad pone al ser humano frente al amor en una actitud de aprendizaje permanente porque "en realidad, todos están sedientos de amor... sin embargo casi nadie piensa que hay algo que aprender acerca del amor" (Fromm, 2007, p. 13). Muchas veces la humanidad camina sumida en el amor posesivo, sentimentalista y egocéntrico que a la larga conduce al vacío interior en medio de supuestas riquezas externas. "Si amo realmente a una persona, amo a todas las personas, amo al mundo, amo a la vida. Si puedo decirle a alguien "te amo", debo poder decir "amo a todos en ti, a través de ti amo al mundo, en ti me amo también a mí mismo” (Fromm, 2007, p. 68).

Por lo tanto, aprender a amar es una tarea permanente puesto que "el hombre es un ser "indigente" y un ser "oferente". Por eso el que se encierra en su soledad intentando ganarse se pierde. Hace falta darse para poder explorar todo lo que somos. La persona se fundamenta en un don, en una entrega. El más inteligente de los hombres no alcanza su plenitud hasta que ama” (Gastaldi-Perrelló, 1996, p. 38). 
El amor que cada uno experiencia más allá del egoísmo, implica el ejercicio de la libertad. Sin libertad no hay amor, pues no se puede obligar a amar a otro. Se ama desde la libertad puesto que la persona es un sujeto libre (Barraca, 2005, p. 28).

Ahora bien, esta capacidad de amar se expresa peculiarmente de forma sexuada, es decir que el ser humano se reconoce a sí mismo perteneciente a lo masculino o femenino y como tal se abre a los demás. El ser varón y ser mujer son dos proyectos existenciales igualmente dignos, pero diferentes que con el telón de fondo del amor pueden provocar el encuentro profundo, la entrega total, la radical comunión interpersonal.

A la comunicación, interacción, encuentro, diálogo y comunión que el ser humano establece con la riqueza del ser varón y ser mujer es lo que llamamos sexualidad. Es "una dimensión desde donde se comprende la totalidad del ser humano y, el ser humano, cuanto espíritu corporeizado experiencia su sexualidad como la riqueza más profunda pero no la única de su ser existente" (Plasencia, 2013, p. 87). La sexualidad es una dimensión abarcante de toda la persona, que invade todo y marca a nivel biológico, psíquico y espiritual; matiza las relaciones humanas y configura la propia personalidad. Todo en el ser humano queda revestido de sexualidad.

En este punto es muy importante enfatizar que la sexualidad no se reduce únicamente a la experiencia del placer, sino que se constituye en una vivencia permanente del don de sí. Hombre y mujer se encuentran con la riqueza de su masculinidad y feminidad en un diálogo de complementariedad y reciprocidad. Si bien existen las diferencias biológicas y psicológicas propias de cada sexo, esto no constituye un obstáculo para la comunión interpersonal, al contrario, son su riqueza más valiosa pues varón y mujer se encuentran y enriquecen en la diferencia. Cada uno contribuye con la riqueza de su ser sexuado para construir un proyecto común. 
En este sentido, la sexualidad y sus signos concretos (besos, caricias, abrazos, miradas, encuentro genital, etc.) se revisten de profundidad cuando son expresión y consecuencia del amor que las personas se profesan, sin amor el sexo se banaliza y se vacía de contenido y significado. De allí que es importante tener en cuenta los niveles en el diálogo del amor: genitalidad (sexo), afectividad (eros) y espiritualidad (ágape).

El nivel de la genitalidad (sexo) se refiere a la atracción sexual biológica, al encuentro genital; aquí nos enamoramos del atractivo físico y biológico que tiene la otra persona. Es el primer nivel del amor y como tal tiene significado en cuanto hace evidente la comunión entre dos personas. El nivel de la afectividad (eros) tiene que ver con la orientación afectiva y el gusto de estar y compartir con el otro ya no tanto en el encuentro genital sino en la demostración de ternura y afecto, es la atracción sexual sicológica; aquí nos enamoramos de los sentimientos, emociones, inteligencia, personalidad; incluso, el tono de voz, la forma de mirar, la forma de caminar, etc., se colman de una carga afectiva. El nivel de la espiritualidad (ágape) se refiere a la comunión profunda entre dos personas que conduce a descubrir la originalidad del otro/a, al respeto profundo de la libertad y del proyecto de vida, al deseo de promover, a buscar la realización, a buscar sentidos y significados. Aquí ya no se ama al otro por lo que tiene sino por lo que es. En este nivel nos enamoramos de las opciones fundamentales, de los ideales, de los valores, del proyecto de vida, de las creencias, de las motivaciones profundas que tiene el otro, es el amor al otro por lo que es y no por lo que tiene (Gastaldi, 1990, pp. 122-123).

Estos tres niveles tienen que estar siempre armonizados y jerarquizados para vivir una auténtica y profunda sexualidad.

\section{Conclusión}

La sexualidad se puede contextualizar como una distribución de diversos actos que el ser humano como tal tiende a necesitar tanto biológico, psicológico, de placer, entre otros. Se puede decir que la 
sexualidad humana puede distinguir las cualidades de sexo, tendencias, inclinaciones, conductas, valores del ser humano.

El ser humano aprende a conocerse a sí mismo en muchas cosas por las necesidades que aparecen en su entorno, tratando de satisfacerlas tanto en la carne como en otros aspectos. El hombre como la mujer construye su estructura mental y su vida diaria como mejor le convenga tomando en cuenta actitudes favorecedoras que permitan cumplir con sus ambiciones y placeres que pueden ser desde disfrutar de una buena comida con amigos o hasta poder convencerse de un acto sexual, si este fuera el caso. En nuestro contexto social cambiante, globalizado y pocas veces formativo se hace imprescindible que este tema sea vaya contribuyendo con nuevos aportes y experiencias que enriquezcan nuestros niños y jóvenes, y porque no a nosotros los adultos que necesitamos de este desarrollo como hemos visto para nuestra realización plena.

\section{Bibliografía}

Azpirante, E. (2001). Simbolismo de la sexualidad humana: criterios para una ética sexual. Santander: Sal Terrae.

Barraca, J. (2005). Una antropología educativa fundada en el amor. Madrid: CCS. Blanco, L. (2013). Ética integral. Bogotá: Ecoe.

Escudero, J. L. (2007). Hacia una fenomenología de los afectos: Martin Heidegger y Max Scheler. Tématha Revista de Filosofía, 39. Barcelona: Universidad Autónoma de Barcelona.

Fernández, L. (2004). Amor y sexualidad: algunos desafíos. Universidades, 21-33. Foucalt, P. M. (Junio de 1982). Sexo, poder y gobierno de la identidad. (t. J. Bauzá, Entrevistador).

Fromm, E. (2007). El arte de amar. Barcelona: Ediciones Paidós Ibérica S.A. García, B. (2009). Las dimensiones afectivas de la docencia. Revista Digital Universitaria, 10(11). UNAM.

Gastaldi, I. (2003). El hombre un misterio. Quito: Abya-Yala.

Gracia, D. (1998). Ética de la sexualidad. En: J. Botella Llusiá y A. Fernández de Molina (Eds.), La evolución de la sexualidad y los estados intersexuales. Madrid: Ediciones Díaz de Santos. 
Hernández, M., y Sánchez, F. (2008). La dimensión afectiva como base del desarrollo humano. Una reflexión teórica para la intervención en trabajo social. Revista Eleuthera, 2, enero- diciembre. Universidad de Caldas.

López Trujillo, A. (2013). Sexualidad humana: verdad y significado. Disponible en: https://goo.gl/tn4X9L

Montoya Montoya, G. (2007). La ética del cuidado en el contexto de la salud sexual y reproductiva. Acta bioethica 13(2), 168-175.

Plasencia, V. (2013). Antropología cristiana. Quito: UPS.

Rius, L. (2001). Amor, sexo y el fin del milenio. Revista Cubana de Pscicología 18(2), 162-176.

Vaticano (1995). Pontificio Consejo para la familia. Sexualidad humana: verdad $y$ significado 20(3), 127-135. 